\title{
CERTAIN FAMILIES OF MULTIVARIABLE CHAN-CHYAN-SRIVASTAVA POLYNOMIALS
}

\author{
M.A. ÖZARSLAN, R. SRIVASTAVA, AND C. KAANOĞLU
}

Received 26 April, 2016

\begin{abstract}
In this paper we introduce $r$-parameter Srivastava polynomials in $r$-variable by inserting new indices. These polynomials include the Lagrange polynomials in several variables, which are also known as Chan-Chyan-Srivastava polynomials (W.-C.C. Chan, C.-J. Chyan and H.M. Srivastava, The Lagrange polynomials in several variables, Integral Transforms and Special Functions, 12 (2001) 139-148). We prove several two sided linear generating relations between $r$-variable and $(r-1)$ - variable Chan-Chyan-Srivastava polynomials. Some special cases of the main results are also presented.
\end{abstract}

2010 Mathematics Subject Classification: 33C45

Keywords: generating functions, Srivastava polynomials, Chan-Chyan-Srivastava polynomials

\section{INTRODUCTION}

In 1972, Srivastava [21] introduced the following family of polynomials,

$$
S_{n}^{N}(z):=\sum_{k=0}^{\left[\frac{n}{N}\right]} \frac{(-n)_{N k}}{k !} A_{n, k} z^{k} \quad\left(n \in \mathbb{N}_{0}=\mathbb{N} \cup\{0\} ; N \in \mathbb{N}\right),
$$

where $\mathbb{N}$ is the set of positive integers, $\left\{A_{n, k}\right\}_{n, k=0}^{\infty}$ is a bounded double sequence of real or complex numbers, $[a]$ denotes the greatest integer in $a \in \mathbb{R}$, and $(\lambda)_{\nu}$, $(\lambda)_{0} \equiv 1$, denotes the Pochhammer symbol defined by

$$
(\lambda)_{v}:=\frac{\Gamma(\lambda+v)}{\Gamma(\lambda)}
$$

by means of familiar Gamma functions. After, González et al. [13] extended the Srivastava polynomials $S_{n}^{N}(z)$ as follows:

$$
S_{n, m}^{N}(z):=\sum_{k=0}^{\left[\frac{n}{N}\right]} \frac{(-n)_{N k}}{k !} A_{n+m, k} z^{k} \quad\left(m, n \in \mathbb{N}_{0} ; N \in \mathbb{N}\right),
$$


and investigated their properties extensively. In [3], the following family of bivariate polynomials has been introduced

$$
S_{n}^{m, N}(x, y)=\sum_{k=0}^{\left[\frac{n}{N}\right]} A_{m+n, k} \frac{x^{n-N k}}{(n-N k) !} \frac{y^{k}}{k !} \quad\left(m, n \in \mathbb{N}_{0}, N \in \mathbb{N}\right),
$$

and it has shown that the polynomials $S_{n}^{m, N}(x, y)$ includes many well known polynomials such as Lagrange-Hermite polynomials, Lagrange polynomials and HermiteKampé de Feriét polynomials (see also [20]). In [23], Srivastava et al. have introduced the three-variable polynomials

$$
\begin{aligned}
& S_{n}^{m, M, N}(x, y, z)=\sum_{k=0}^{\left[\frac{n}{N}\right]} \sum_{l=0}^{\left[\frac{k}{M}\right]} A_{m+n, k, l} \frac{x^{l}}{l !} \frac{y^{k-M l}}{(k-M l) !} \frac{z^{n-N k}}{(n-N k) !} \\
& \left(m, n \in \mathbb{N}_{0} ; M, N \in \mathbb{N}\right) \text {, }
\end{aligned}
$$

where $\left\{A_{m, n, k}\right\}$ be a triple sequence of complex numbers. Suitable choices of $\left\{A_{m, n, k}\right\}$ in equation (1) gives three variable version of well-known polynomials (see also [14]). In [15], the multivariable extension of the Srivastava polynomials in $r$-variable was introduced:

$$
\begin{gathered}
\sum_{k_{r-1}=0} \sum_{k_{r-2}=0}^{S_{n}^{m}, N_{1}, N_{2}, \ldots, N_{r-1}}\left(x_{1}, x_{2}, \ldots, x_{r}\right) \\
\left.\sum_{k_{1}=0}^{N_{r-2}}\right] \sum_{\left(m, n \in \mathbb{N}_{0} ; N_{1}, N_{2}, \ldots, N_{r-1} \in \mathbb{N}\right),}^{\left[\frac{k_{2}}{N_{1}}\right]} A_{m+n, k_{r-1}, k_{1}, \ldots, k_{r-2}} \frac{x_{1}^{k_{1}}}{k_{1} !} \frac{x_{2}^{k_{2}-N_{1} k_{1}}}{\left(k_{2}-N_{1} k_{1}\right) !} \ldots \frac{x_{r}^{n-N_{r-1} k_{r-1}}}{\left(n-N_{r-1} k_{r-1}\right) !}
\end{gathered}
$$

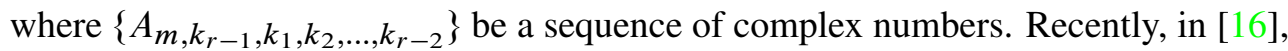
two-parameter one-variable Srivastava polynomials

$$
\begin{gathered}
S_{n}^{m_{1}, m_{2}}(x):=\sum_{\substack{k=0 \\
\left(m_{1}, m_{2}, n, k \in \mathbb{N}_{0}\right),}}^{n} \frac{(-n)_{k}}{k !} A_{m_{1}+m_{2}+n, m_{2}+k} x^{k}
\end{gathered}
$$

two-parameter two-variable Srivastava polynomials

$$
\begin{gathered}
S_{n}^{m_{1}, m_{2}}(x, y)=\sum_{\substack{k=0 \\
\left(m_{1}, m_{2}, n, k \in \mathbb{N}_{0}\right),}}^{n} A_{m_{1}+m_{2}+n, m_{2}+k} \frac{x^{k}}{k !} \frac{y^{n-k}}{(n-k) !} \\
\end{gathered}
$$


and two-parameter three-variable Srivastava polynomials

$$
\begin{aligned}
S_{n}^{m_{1}, m_{2}, M}(x, y, z)= & \sum_{\substack{k=0 \\
\left(m_{1}, m_{2}, n, k, l \in \mathbb{N}_{0}, M \in \mathbb{N}\right)}}^{\left[\frac{k}{M}\right]} A_{m_{1}+m_{2}+n, m_{2}+k, l} \frac{x^{l}}{l !} \frac{y^{k-M l}}{(k-M l) !} \frac{z^{n-k}}{(n-k) !}
\end{aligned}
$$

were introduced. These polynomials include the family of polynomials which were introduced or investigated in $[3,13,15,17,19,21,23]$. In this paper we introduce $r$-parameter Srivastava polynomials in $r$-variables by inserting new indices. These polynomials include the Lagrange polynomials in several variables, which are also known as Chan-Chyan-Srivastava polynomials [4]. We prove several two sided linear generating relations and obtain various generating relations for the polynomials.

\section{R-PARAMETER R-VARIABLE SRIVASTAVA POLYNOMIALS}

In this section we define $r$-parameter $r$-variable Srivastava polynomials as follows:

$$
\begin{gathered}
S_{n}^{m_{1}, m_{2}, \ldots, m_{r-1}, M}\left(x_{1}, x_{2}, \ldots, x_{r}\right) \\
\sum_{k_{r-1}=0}^{n} \sum_{k_{r-2}=0}^{k_{r-1}} \ldots \sum_{\substack{k_{2}=0 \\
\left(m_{1}, m_{2}, \ldots, m_{r-1}, n \in \mathbb{N}_{0} ; M \in \mathbb{N}\right),}}^{k_{3}=0} \frac{x_{1}^{k_{1}}}{k_{1} !} \frac{x_{2}^{k_{2}-M k_{1}}}{\left(k_{2}-M k_{1}\right) !} \ldots \frac{x_{r}^{n-k_{r-1}}}{\left(n-k_{r-1}\right) !},
\end{gathered}
$$

where $\Omega=m_{1}+m_{2}+\ldots+m_{r-1}+n, m_{2}+\ldots+m_{r-1}+k_{r-1}, k_{1}, m_{r-1}+k_{2}$, $m_{r-2}+m_{r-1}+k_{3}, \ldots, m_{3}+\ldots+m_{r-1}+k_{r-2}$. Note that appropriate choices of the

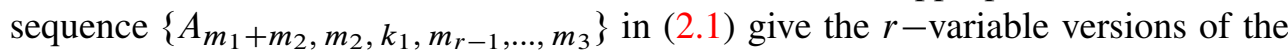
well known polynomials.

Remark 1. The Lagrange polynomials in several variables, which are known as Chan-Chyan-Srivastava polynomials [4] are defined by the generating function relation

$$
\begin{aligned}
& \prod_{j=1}^{r}\left(1-x_{j} t\right)^{-\alpha_{j}}=\sum_{n=0}^{\infty} g_{n}^{\left(\alpha_{1}, \alpha_{2}, \ldots, \alpha_{r}\right)}\left(x_{1}, x_{2}, \ldots, x_{r}\right) t^{n} \\
& \left(\alpha_{j} \in \mathbb{C}(j=1, \ldots, r) ;|t|<\min \left\{\left|x_{1}\right|^{-1}, \ldots,\left|x_{r}\right|^{-1}\right\}\right) .
\end{aligned}
$$

The polynomials are given explicitly by

$$
\begin{aligned}
& g_{n}^{\left(\alpha_{1}, \alpha_{2}, \ldots, \alpha_{r}\right)}\left(x_{1}, x_{2}, \ldots, x_{r}\right) \\
& =\sum_{k_{r-1}=0}^{n} \ldots \sum_{k_{2}=0}^{k_{3}} \sum_{k_{1}=0}^{k_{2}}\left(\alpha_{1}\right)_{k_{1}}\left(\alpha_{2}\right)_{k_{2}-k_{1}} \ldots\left(\alpha_{r-1}\right)_{k_{r-1}-k_{r-2}}\left(\alpha_{r}\right)_{n-k_{r-1}}
\end{aligned}
$$




$$
\times \frac{x_{1}^{k_{1}}}{k_{1} !} \frac{x_{2}^{k_{2}-k_{1}}}{\left(k_{2}-k_{1}\right) !} \ldots \frac{x_{r-1}^{k_{r-1}-k_{r-2}}}{\left(k_{r-1}-k_{r-2}\right) !} \frac{x_{r}^{n-k_{r-1}}}{\left(n-k_{r-1}\right) !} .
$$

Many authors have studied the properties of these polynomials. For example, the bilateral generating functions for these polynomials and miscellaneous properties are given in Liu et al. [12,18]. In [8], the orthogonality properties and various integral representations for these polynomials are given (see also [1,2,5-7]). Furthermore, these polynomials are used in approximation theory. In [11], Duman et al. investigated some approximation properties of positive linear operators constructed by these polynomials (see also [9, 10]). In [22] Srivastava et al. investigated umbral calculus presentations of these polynomials.

Remark 2. If we set $M=1$ and

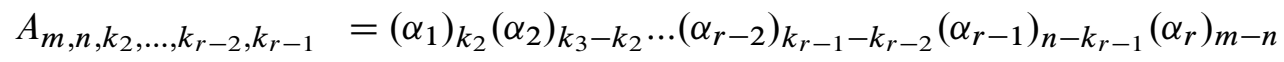

in equation (2.1), we find that

$$
\begin{aligned}
& A_{m_{1}+\ldots+m_{r-1}+n, m_{2}+\ldots+m_{r-1}+k_{r-1}, k_{1}, m_{r-1}+k_{2}, m_{r-2}+m_{r-1}+k_{3}, \ldots, m_{3}+\ldots+m_{r-1}+k_{r-2}} \\
& =\left(\alpha_{1}\right)_{k_{1}}\left(\alpha_{2}\right)_{m_{r-1}+k_{2}-k_{1}}\left(\alpha_{3}\right)_{m_{r-2}}+k_{3}-k_{2} \ldots\left(\alpha_{r-1}\right)_{m_{2}+k_{r-1}-k_{r-2}}\left(\alpha_{r}\right)_{m_{1}+n-k_{r-1}} \\
& =\left(\alpha_{2}\right)_{m_{r-1}}\left(\alpha_{3}\right)_{m_{r-2}} \ldots\left(\alpha_{r-1}\right)_{m_{2}}\left(\alpha_{r}\right)_{m_{1}}\left(\alpha_{1}\right)_{k_{1}}\left(\alpha_{2}+m_{r-1}\right)_{k_{2}-k_{1}}\left(\alpha_{3}+m_{r-2}\right)_{k_{3}-k_{2}} \\
& \times \ldots\left(\alpha_{r-1}+m_{2}\right)_{k_{r-1}-k_{r-2}}\left(\alpha_{r}+m_{1}\right)_{n-k_{r-1}}
\end{aligned}
$$

and therefore

$$
\begin{aligned}
& S_{n}^{m_{1}, m_{2}, \ldots, m_{r-1}, 1}\left(x_{1}, x_{2}, \ldots, x_{r}\right)= \\
& \left(\alpha_{2}\right)_{m_{r-1}}\left(\alpha_{3}\right)_{m_{r-2}} \ldots\left(\alpha_{r-1}\right)_{m_{2}}\left(\alpha_{r}\right)_{m_{1}} g_{n}^{\left(\alpha_{1}, \alpha_{2}+m_{r-1}, \alpha_{3}+m_{r-2}, \ldots, \alpha_{r}+m_{1}\right)}\left(x_{1}, \ldots, x_{r}\right) .
\end{aligned}
$$

Now, let recall an infinite series identities which were obtained by Srivastava et al. [23].

Lemma 1 (see [23], Lemma 1). Let $N_{1}, N_{2}, \ldots, N_{r-1} \in \mathbb{N}, r=\{2,3, \ldots\}$. Then

$$
\begin{aligned}
& \sum_{n_{r}=0}^{\infty} \sum_{n_{r-1}=0}^{\infty} \ldots \sum_{n_{1}=0}^{\infty} A\left(n_{1}, n_{2}, \ldots, n_{r}\right) \\
& =\sum_{n_{r}=0}^{\infty} \sum_{n_{r-1}=0}^{\left[\frac{n_{r}}{N_{r-1}}\right]} \ldots \sum_{n_{1}=0}^{\left[\frac{n_{2}}{N_{1}}\right]} A\left(n_{1}, n_{2}-N_{1} n_{1}, \ldots, n_{r}-N_{r-1} n_{r-1}\right)
\end{aligned}
$$

and

$$
\sum_{n_{r}=0}^{\infty} \sum_{n_{r-1}=0}^{\left[\frac{n_{r}}{N_{r-1}}\right]} \ldots \sum_{n_{1}=0}^{\left[\frac{n_{2}}{N_{1}}\right]} A\left(n_{1}, n_{2}, \ldots, n_{r}\right)
$$




$$
\begin{aligned}
& =\sum_{n_{r}=0}^{\infty} \sum_{r_{r-1}=0}^{\infty} \ldots \sum_{n_{1}=0}^{\infty} A\left(n_{1}, n_{2}+N_{1} n_{1}, n_{3}+N_{2} N_{1} n_{1}+N_{2} n_{2}, \ldots,\right. \\
& \left.n_{r}+\prod_{j=1}^{r-1} N_{j} n_{1}+\prod_{j=2}^{r-1} N_{j} n_{2}+\ldots+N_{r-1} n_{r-1}\right)
\end{aligned}
$$

where $\left\{A\left(n_{1}, n_{2}, \ldots, n_{r}\right)\right\}$ is a bounded $r$-tuple sequence of real or complex numbers.

The main result of this paper is given by the following theorem.

Theorem 1. Let $\{f(n)\}_{n=0}^{\infty}$ be a bounded sequence of complex numbers. Then

$$
\begin{aligned}
& \sum_{m_{1}, \ldots, m_{r-1}, n=0}^{\infty} f\left(m_{1}+\ldots+m_{r-1}+n\right) \\
\times & S_{n}^{m_{1}, m_{2}, \ldots, m_{r-1}, M}\left(x_{1}, x_{2}, \ldots, x_{r}\right) \frac{w_{1}^{m_{1}}}{m_{1} !} \ldots \frac{w_{r-1}^{m_{r-1}}}{m_{r-1} !} t^{n} \\
= & \sum_{k_{1}, m_{1}, \ldots, m_{r-1}=0}^{\infty} f\left(m_{1}+\ldots+m_{r-1}+M k_{1}\right) \\
\times & A_{m_{1}+m_{2}+\ldots+m_{r-1}+M k_{1}, m_{2}+\ldots+m_{r-1}+M k_{1}, k_{1}, m_{r-1}+M k_{1}, \ldots, m_{3}+\ldots+m_{r-1}+M k_{1}} \\
\times & \frac{\left(x_{1} t^{M}\right)^{k_{1}}}{k_{1} !} \frac{\left(x_{r} t+w_{1}\right)^{m_{1}}}{m_{1} !} \frac{\left(x_{r-1} t+w_{2}\right)^{m_{2}}}{m_{2} !} \ldots \frac{\left(x_{2} t+w_{r-1}\right)^{m_{r-1}}}{m_{r-1} !}
\end{aligned}
$$

provided that each member of the series identity (2.2) exists.

Proof. Let the left hand side of (2.2) be denoted by $\Psi\left(x_{1}, \ldots, x_{r}\right)$. Then using the definition of $S_{n}^{m_{1}, m_{2}, \ldots, m_{r-1}, M}\left(x_{1}, x_{2}, \ldots, x_{r}\right)$ on the left hand side of (2.2), we have

$$
\begin{aligned}
& \Psi\left(x_{1}, \ldots, x_{r}\right) \\
& =\sum_{m_{1}, \ldots, m_{r-1}, n=0}^{\infty} f\left(m_{1}+\ldots+m_{r-1}+n\right) \\
& \times \sum_{k_{r-1}=0}^{n} \sum_{k_{r-2}=0}^{k_{r-1}} \ldots \sum_{k_{2}=0}^{k_{3}} \sum_{k_{1}=0}^{\left[\frac{k_{2}}{M}\right]} A_{\Omega} \frac{x_{1}^{k_{1}}}{k_{1} !} \frac{x_{2}^{k_{2}-M k_{1}}}{\left(k_{2}-M k_{1}\right) !} \ldots \frac{x_{r}^{n-k_{r-1}}}{\left(n-k_{r-1}\right) !} \frac{w_{1}^{m_{1}}}{m_{1} !} \ldots \frac{w_{r-1}^{m_{r-1}}}{m_{r-1} !} t^{n} .
\end{aligned}
$$

Let define

$$
F(r-1):=M k_{1}+k_{2}+k_{3}+\ldots+k_{r-1} .
$$

By applying Lemma 1, we find

$$
\Psi\left(x_{1}, \ldots, x_{r}\right)
$$




$$
\begin{aligned}
& =\sum_{k_{1}, \ldots, k_{r-1}, m_{1}, \ldots, m_{r-1}, n=0}^{\infty} f\left(m_{1}+\ldots+m_{r-1}+n+F(r-1)\right) \\
& \times A_{\Omega_{1}} \frac{x_{1}^{k_{1}}}{k_{1} !} \ldots \frac{x_{r-1}^{k_{r-1}}}{k_{r-1} !} \frac{x_{r}^{n}}{n !} \frac{w_{1}^{m_{1}}}{m_{1} !} \ldots \frac{w_{r-1}^{m_{r-1}}}{m_{r-1} !} t^{n+M k_{1}+k_{2}+\ldots+k_{r-1}}
\end{aligned}
$$

where $\Omega_{1}=m_{1}+m_{2}+\ldots+m_{r-1}+n+F(r-1), m_{2}+\ldots+m_{r-1}+k_{r-1}+F(r-$ 2),

$k_{1}, m_{r-1}+k_{2}+M k_{1}, \ldots, m_{3}+\ldots+m_{r-1}+k_{r-2}+F(r-3)$.

Let $m_{1} \rightarrow m_{1}-n, m_{2} \rightarrow m_{2}-k_{r-1}, m_{3} \rightarrow m_{3}-k_{r-2}$,

.., $m_{r-2} \rightarrow m_{r-2}-k_{3}, m_{r-1} \rightarrow m_{r-1}-k_{2}$ then

$$
\begin{aligned}
& \Psi\left(x_{1}, \ldots, x_{r}\right) \\
& =\sum_{k_{1}, m_{1}, \ldots, m_{r-1}=0}^{\infty} f\left(m_{1}+\ldots+m_{r-1}+M k_{1}\right) \\
& \times A_{m_{1}+\ldots+m_{r-1}+M k_{1}, m_{2}+\ldots+m_{r-1}+M k_{1}, k_{1}, m_{r-1}+M k_{1}, \ldots, m_{3}+\ldots+m_{r-1}+M k_{1}} \\
& \times \frac{\left(x_{1} t^{M}\right)^{k_{1}}}{k_{1} !} \frac{\sum_{n=0}^{m_{1}}\left(\begin{array}{c}
m_{1} \\
n
\end{array}\right)\left(x_{r} t\right)^{n} w_{1}^{m_{1}-n}}{m_{1} !} \sum_{k_{r-1}=0}^{m_{2}}\left(\begin{array}{c}
m_{2} \\
k_{r-1}
\end{array}\right)\left(x_{r-1} t\right)^{k_{r-1}} w_{2}^{m_{2}-k_{r-1}} \\
& m_{2} ! \\
& \times \ldots \frac{\sum_{k_{2}=0}^{m_{r-1}}\left(\begin{array}{c}
m_{r-1} \\
k_{2}
\end{array}\right)\left(x_{2} t\right)^{k_{2}} w_{r-1}^{m_{r-1}-k_{2}}}{m_{r-1} !}
\end{aligned}
$$

and

$$
\begin{aligned}
& \Psi\left(x_{1}, \ldots, x_{r}\right) \\
= & \sum_{k_{1}, m_{1}, \ldots, m_{r-1}=0}^{\infty} f\left(m_{1}+\ldots+m_{r-1}+M k_{1}\right) \\
\times & A_{m_{1}+m_{2}+\ldots+m_{r-1}+M k_{1}, m_{2}+\ldots+m_{r-1}+M k_{1}, k_{1}, m_{r-1}+M k_{1}, \ldots, m_{3}+\ldots+m_{r-1}+M k_{1}} \\
\times & \frac{\left(x_{1} t^{M}\right)^{k_{1}}}{k_{1} !} \frac{\left(x_{r} t+w_{1}\right)^{m_{1}}}{m_{1} !} \frac{\left(x_{r-1} t+w_{2}\right)^{m_{2}}}{m_{2} !} \ldots \frac{\left(x_{2} t+w_{r-1}\right)^{m_{r-1}}}{m_{r-1} !} .
\end{aligned}
$$

Whence the result.

Corollary 1. Let the polynomials $S_{n}^{m_{1}, m_{2}, \ldots, m_{r-1}, M}\left(x_{1}, x_{2}, \ldots, x_{r}\right)$ be defined by equation (2.1). Suppose also that $r-1$-variable polynomials $P_{m_{2}}^{M}\left(x_{1}, \ldots, x_{r-1}\right)$ are defined by

$$
\begin{aligned}
& P_{m_{2}}^{M}\left(x_{1}, \ldots, x_{r-1}\right) \\
& =\sum_{m_{3}=0}^{m_{2}} \ldots \sum_{m_{r-1}=0}^{m_{r-2}} \sum_{k_{1}=0}^{\left[\frac{m_{r-1}}{M}\right]} A_{m_{1}+m_{2}, m_{2}, k_{1}, m_{r-1}, \ldots, m_{3}}
\end{aligned}
$$




$$
\times \frac{\left(x_{1}\right)^{k_{1}}}{k_{1} !} \frac{\left(x_{2}\right)^{m_{r-1}-M k_{1}}}{\left(m_{r-1}-M k_{1}\right) !} \frac{\left(x_{3}\right)^{m_{r-2}-m_{r-1}}}{\left(m_{r-2}-m_{r-1}\right) !} \ldots \frac{\left(x_{r-1}\right)^{m_{2}-m_{3}}}{\left(m_{2}-m_{3}\right) !} .
$$

Then, for a suitably bounded sequence $\{f(n)\}_{n=0}^{\infty}$, the following family of twosided linear generating relations holds true between the $r$-variable polynomials $S_{n}^{m_{1}, m_{2}, \ldots, m_{r-1}, M}\left(x_{1}, \ldots, x_{r}\right)$ and $(r-1)-$ variable polynomials $P_{m_{2}}^{M}\left(x_{1}, \ldots, x_{r-1}\right)$ :

$$
\begin{aligned}
& \sum_{m_{1}, \ldots, m_{r-1}, n=0}^{\infty} f\left(m_{1}+\ldots+m_{r-1}+n\right) S_{n}^{m_{1}, \ldots, m_{r-1}, M}\left(x_{1}, \ldots, x_{r}\right) \frac{w_{1}^{m_{1}}}{m_{1} !} \ldots \frac{w_{r-1}^{m_{r-1}}}{m_{r-1} !} t^{n} \\
= & \sum_{m_{1}, m_{2}=0}^{\infty} f\left(m_{1}+m_{2}\right) \frac{\left(x_{r} t+w_{1}\right)^{m_{1}}}{m_{1} !} P_{m_{2}}^{M}\left(x_{1} t^{M}, x_{2} t+w_{r-1}, \ldots, x_{r-1} t+w_{2}\right)
\end{aligned}
$$

provided that each member of the series identity (2.4) exists.

Proof. If we set $m_{r-1} \rightarrow m_{r-1}-M k_{1}, m_{r-2} \rightarrow m_{r-2}-m_{r-1}$, $\ldots, m_{2} \rightarrow m_{2}-m_{3}$ in the right side of the equation (2.2) then

$$
\begin{aligned}
& \sum_{m_{1}, \ldots, m_{r-1}, n=0}^{\infty} f\left(m_{1}+\ldots+m_{r-1}+n\right) S_{n}^{m_{1}, \ldots, m_{r-1}, M}\left(x_{1}, \ldots, x_{r}\right) \frac{w_{1}^{m_{1}}}{m_{1} !} \ldots \frac{w_{r-1}^{m_{r-1}}}{m_{r-1} !} t^{n} \\
= & \sum_{m_{1}, m_{2}=0}^{\infty} f\left(m_{1}+m_{2}\right) \frac{\left(x_{r} t+w_{1}\right)^{m_{1}}}{m_{1} !} \\
\times & \sum_{m_{3}=0}^{m_{2}} \ldots \sum_{m_{r-1}=0}^{m_{r-2}} \sum_{k_{1}=0}^{\left[\frac{m_{r-1}}{M}\right]} A_{m_{1}+m_{2}, m_{2}, k_{1}, m_{r-1}, \ldots, m_{3}} \frac{\left(x_{1} t^{M}\right)^{k_{1}}}{k_{1} !} \\
\times & \frac{\left(x_{2} t+w_{r-1}\right)^{m_{r-1}-M k_{1}}}{\left(m_{r-1}-M k_{1}\right) !} \frac{\left(x_{3} t+w_{r-2}\right)^{m_{r-2}-m_{r-1}}}{\left(m_{r-2}-m_{r-1}\right) !} \ldots \frac{\left(x_{r-1} t+w_{2}\right)^{m_{2}-m_{3}}}{\left(m_{2}-m_{3}\right) !} \\
= & \sum_{m_{1}, m_{2}=0}^{\infty} f\left(m_{1}+m_{2}\right) \frac{\left(x_{r} t+w_{1}\right)^{m_{1}}}{m_{1} !} P_{m_{2}}\left(x_{1} t^{M}, x_{2} t+w_{r-1}, \ldots, x_{r-1} t+w_{2}\right) .
\end{aligned}
$$

By setting $t=-\frac{w_{1}}{x_{r}}$ in (2.4), we get the following result.

Corollary 2. Under the hypotheses of Corollary 1, we get the following generating relation

$$
\sum_{m_{1}, \ldots, m_{r-1}, n=0}^{\infty} f\left(m_{1}+\ldots+m_{r-1}+n\right)
$$




$$
\begin{aligned}
& \times S_{n}^{m_{1}, \ldots, m_{r-1}, M}\left(x_{1}, \ldots, x_{r}\right) \frac{w_{1}^{m_{1}}}{m_{1} !} \ldots \frac{w_{r-1}^{m_{r-1}}}{m_{r-1} !}\left(-\frac{w_{1}}{x_{r}}\right)^{n} \\
& =\sum_{m_{2}=0}^{\infty} f\left(m_{2}\right) P_{m_{2}}^{M}\left(x_{1}\left(-\frac{w_{1}}{x_{r}}\right)^{M}, x_{2}\left(-\frac{w_{1}}{x_{r}}\right)+w_{r-1}, \ldots, x_{r-1}\left(-\frac{w_{1}}{x_{r}}\right)+w_{2}\right) .
\end{aligned}
$$

Remark 3. Choosing $M=1$ and

$$
A_{m, n, k_{2}, k_{3}, \ldots, k_{r-1}}=\left(\alpha_{1}\right)_{k_{2}}\left(\alpha_{2}\right)_{k_{3}-k_{2}} \ldots\left(\alpha_{r-1}\right)_{n-k_{r-1}}\left(\alpha_{r}\right)_{m-n}
$$

in (2.3) we observe that

$$
\begin{aligned}
& A_{m_{1}+m_{2}, m_{2}, k_{1}, m_{r-1}, m_{r-2}, \ldots, m_{4}, m_{3}}
\end{aligned}
$$

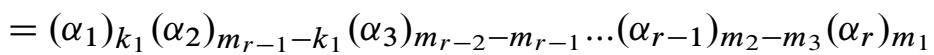

then

$$
P_{n}^{1}\left(x_{1}, \ldots, x_{r-1}\right)=\left(\alpha_{r}\right)_{m_{1}} g_{n}^{\left(\alpha_{1}, \alpha_{2}, \ldots, \alpha_{r-1}\right)}\left(x_{1}, x_{2}, \ldots, x_{r-1}\right)
$$

where $g_{n}^{\left(\alpha_{1}, \alpha_{2}, \ldots, \alpha_{r-1}\right)}\left(x_{1}, x_{2}, \ldots, x_{r-1}\right)$ is the Lagrange polynomials of $(r-1)$-variables.

Hence, upon setting $M=1$ and considering (2.5) and (2.7) in Corollary 2, we get

$$
\begin{aligned}
& \sum_{n=0}^{\infty} f(n) g_{n}^{\left(\alpha_{1}, \alpha_{2}, \ldots, \alpha_{r-1}\right)}\left(x_{1}\left(-\frac{w_{1}}{x_{r}}\right), x_{2}\left(-\frac{w_{1}}{x_{r}}\right)+w_{r-1}, \ldots, x_{r-1}\left(-\frac{w_{1}}{x_{r}}\right)+w_{2}\right) \\
&=\sum_{m_{1}, \ldots, m_{r-1}, n=0}^{\infty} f\left(m_{1}+\ldots+m_{r-1}+n\right)\left(\alpha_{2}\right)_{m_{r-1}}\left(\alpha_{3}\right)_{m_{r-2} \ldots .}\left(\alpha_{r-1}\right)_{m_{2}}\left(\alpha_{r}\right)_{m_{1}} \\
& \times g_{n}^{\left(\alpha_{1}, \alpha_{2}+m_{r-1}, \alpha_{3}+m_{r-2}, \ldots, \alpha_{r}+m_{1}\right)}\left(x_{1}, x_{2}, \ldots, x_{r}\right) \frac{w_{1}^{m_{1}}}{m_{1} !} \ldots \frac{w_{r-1}^{m_{r-1}}}{m_{r-1} !}\left(-\frac{w_{1}}{x_{r}}\right)^{n} .
\end{aligned}
$$

Let choose $f(n)=1$ in (2.7) and consider Remark 1 then we have

$$
\begin{aligned}
& \sum_{n=0}^{\infty} g_{n}^{\left(\alpha_{1}, \alpha_{2}, \ldots, \alpha_{r-1}\right)}\left(x_{1}\left(-\frac{w_{1}}{x_{r}}\right), x_{2}\left(-\frac{w_{1}}{x_{r}}\right)+w_{r-1}, \ldots, x_{r-1}\left(-\frac{w_{1}}{x_{r}}\right)+w_{2}\right) \\
& =\sum_{m_{1}, \ldots, m_{r-1}, n=0}^{\infty} \sum_{k_{r-1}=0}^{n} \ldots \sum_{k_{2}=0}^{k_{3}} \sum_{k_{1}=0}^{k_{2}}\left(\alpha_{2}\right)_{m_{r-1}}\left(\alpha_{3}\right)_{m_{r-2}} \ldots\left(\alpha_{r-1}\right)_{m_{2}}\left(\alpha_{r}\right)_{m_{1}} \\
& \times\left(\alpha_{1}\right)_{k_{1}}\left(\alpha_{2}+m_{r-1}\right)_{k_{2}-k_{1}} \ldots\left(\alpha_{r-1}+m_{2}\right)_{k_{r-1}-k_{r-2}}\left(\alpha_{r}+m_{1}\right)_{n-k_{r-1}} \\
& \times \frac{x_{1}^{k_{1}}}{k_{1} !} \frac{x_{2}^{k_{2}-k_{1}}}{\left(k_{2}-k_{1}\right) !} \ldots \frac{x_{r-1}^{k_{r-1}-k_{r-2}}}{\left(k_{r-1}-k_{r-2}\right) !} \frac{x_{r}^{n-k_{r-1}}}{\left(n-k_{r-1}\right) !} \frac{w_{1}^{m_{1}}}{m_{1} !} \ldots \frac{w_{r-1}^{m_{r-1}}}{m_{r-1} !}\left(-\frac{w_{1}}{x_{r}}\right)^{n} \text {. }
\end{aligned}
$$


Let $\Phi$ denote the right side of (2.8). Applying Lemma 1 into the right side of the equation (2.8) we get

$$
\begin{aligned}
& \Phi=\sum_{\substack{k_{1}, \ldots, k_{r-1}, m_{1}, \ldots, m_{r-1}, n=0 \\
\left(\alpha_{r}\right)_{m_{1}}\left(\alpha_{r-1}\right)_{m_{2}} \ldots\left(\alpha_{3}\right)_{m_{r-2}}\left(\alpha_{2}\right)_{m_{r-1}}\left(\alpha_{r}+m_{1}\right)_{n}}}^{\infty}\left(\alpha_{1}\right)_{k_{1}}\left(\alpha_{2}+m_{r-1}\right)_{k_{2}} \ldots\left(\alpha_{r-1}+m_{2}\right)_{k_{r-1}} \\
& \times \frac{\left(\left(-\frac{w_{1}}{x_{r}}\right) x_{1}\right)^{k_{1}}}{k_{1} !} \frac{\left(\left(-\frac{w_{1}}{x_{r}}\right) x_{2}\right)^{k_{2}}}{k_{2} !} \ldots \frac{\left(\left(-\frac{w_{1}}{x_{r}}\right) x_{r-1}\right)^{k_{r-1}}}{k_{r-1} !} \frac{\left(\left(-\frac{w_{1}}{x_{r}}\right) x_{r}\right)^{n}}{n !} \frac{w_{1}^{m_{1}}}{m_{1} !} \ldots \frac{w_{r-1}^{m_{r-1}}}{m_{r-1} !}
\end{aligned}
$$

and

$$
\begin{aligned}
& \Phi=\sum_{m_{1}, \ldots, m_{r-1}=0}^{\infty}\left(\alpha_{r}\right)_{m_{1}}\left(\alpha_{r-1}\right)_{m_{2}} \ldots\left(\alpha_{3}\right)_{m_{r-2}}\left(\alpha_{2}\right)_{m_{r-1}} \\
& \times\left(1+\left(\frac{w_{1}}{x_{r}}\right) x_{1}\right)^{-\alpha_{1}}\left(1+\left(\frac{w_{1}}{x_{r}}\right) x_{2}\right)^{-\left(\alpha_{2}+m_{r-1}\right)} \ldots\left(1+\left(\frac{w_{1}}{x_{r}}\right) x_{r-1}\right)^{-\left(\alpha_{r-1}+m_{2}\right)} \\
& \times\left(1+\left(\frac{w_{1}}{x_{r}}\right) x_{r}\right)^{-\left(\alpha_{r}+m_{1}\right)} \frac{w_{1}^{m_{1}}}{m_{1} !} \ldots \frac{w_{r-1}^{m_{r-1}}}{m_{r-1} !} \\
& =\left(1+\left(\frac{w_{1}}{x_{r}}\right) x_{1}\right)^{-\alpha_{1}}\left(1+\left(\frac{w_{1}}{x_{r}}\right) x_{2}\right)^{-\alpha_{2}} \ldots\left(1+\left(\frac{w_{1}}{x_{r}}\right) x_{r-1}\right)^{-\alpha_{r-1}}\left(1+\left(\frac{w_{1}}{x_{r}}\right) x_{r}\right)^{-\alpha_{r}} \\
& \times \sum_{m_{1}, \ldots, m_{r-1}=0}^{\infty}\left(\alpha_{r}\right)_{m_{1}}\left(\alpha_{r-1}\right)_{m_{2} \ldots} \ldots\left(\alpha_{3}\right)_{m_{r-2}}\left(\alpha_{2}\right)_{m_{r-1}} \\
& \times\left(1+\left(\frac{w_{1}}{x_{r}}\right) x_{2}\right)^{-m_{r-1}} \ldots\left(1+\left(\frac{w_{1}}{x_{r}}\right) x_{r-1}\right)^{-m_{2}}\left(1+\left(\frac{w_{1}}{x_{r}}\right) x_{r}\right)^{-m_{1}} \frac{w_{1}^{m_{1}}}{m_{1} !} \ldots \frac{w_{r-1}^{m_{r-1}}}{m_{r-1} !} \\
& =\left(1+\left(\frac{w_{1}}{x_{r}}\right) x_{1}\right)^{-\alpha_{1}}\left(1+\left(\frac{w_{1}}{x_{r}}\right) x_{2}\right)^{-\alpha_{2}} \ldots\left(1+\left(\frac{w_{1}}{x_{r}}\right) x_{r-1}\right)^{-\alpha_{r-1}}\left(1+\left(\frac{w_{1}}{x_{r}}\right) x_{r}\right)^{-\alpha_{r}} \\
& \times\left(1-\frac{w_{1}}{1+\left(\frac{w_{1}}{x_{r}}\right) x_{r}}\right)^{-\alpha_{r}}\left(1-\frac{w_{2}}{1+\left(\frac{w_{1}}{x_{r}}\right) x_{r-1}}\right)^{-\alpha_{r-1}} \ldots\left(1-\frac{w_{r-1}}{1+\left(\frac{w_{1}}{x_{r}}\right) x_{2}}\right)^{-\alpha_{2}} \\
& =\left(1+\left(\frac{w_{1}}{x_{r}}\right) x_{1}\right)^{-\alpha_{1}}\left(1+\left(\frac{w_{1}}{x_{r}}\right) x_{2}-w_{r-1}\right)^{-\alpha_{2}} \ldots\left(1+\left(\frac{w_{1}}{x_{r}}\right) x_{r-1}-w_{2}\right)^{-\alpha_{r-1}} \\
& \times\left(1+\left(\frac{w_{1}}{x_{r}}\right) x_{r}-w_{1}\right)^{-\alpha_{r}} .
\end{aligned}
$$

Corollary 3. The generating relation

$$
\begin{aligned}
& \sum_{n=0}^{\infty} g_{n}^{\left(\alpha_{1}, \alpha_{2}, \ldots, \alpha_{r-1}\right)}\left(x_{1}\left(-\frac{w_{1}}{x_{r}}\right), x_{2}\left(-\frac{w_{1}}{x_{r}}\right)+w_{r-1}, \ldots, x_{r-1}\left(-\frac{w_{1}}{x_{r}}\right)+w_{2}\right) \\
& =\left(1+\left(\frac{w_{1}}{x_{r}}\right) x_{1}\right)^{-\alpha_{1}}\left(1+\left(\frac{w_{1}}{x_{r}}\right) x_{2}-w_{r-1}\right)^{-\alpha_{2}} \ldots\left(1+\left(\frac{w_{1}}{x_{r}}\right) x_{r-1}-w_{2}\right)^{-\alpha_{r-1}}
\end{aligned}
$$


is given for $g_{n}^{\left(\alpha_{1}, \alpha_{2}, \ldots, \alpha_{r-1}\right)}\left(x_{1}, x_{2}, \ldots, x_{r-1}\right)$.

\section{REFERENCES}

[1] R. Aktas, R. Sahin, and A. Altin, "On a multivariable extension of Humbert polynomials." Appl. Math. and Comp., vol. 218, no. 3, pp. 662-666, 2011, doi: 10.1016/j.amc.2011.01.082.

[2] A. Altin, R. Aktas, and E. Erkus-Duman, "On a multivariable extension for the extended Jacobi polynomials." Journal of Mathematical Analysis and Applications, vol. 353, no. 1, pp. 121-133, 2009, doi: 10.1016/j.jmaa.2008.11.070.

[3] A. Altin, E. Erkus, and M. Özarslan, "Families of linear generating functions for polynomials in two variables." Integral Transforms and Spec. Funct., vol. 5, no. 17, pp. 315-320, 2006, doi: 10.1080/10652460600568162.

[4] W.-C. Chan, C.-J. Chyan, and H. Srivastava, "The Lagrange polynomials in several variables." Integral Transforms and Spec. Funct., vol. 12, pp. 139-148, 2001, doi: 10.1080/10652460108819340.

[5] K.-Y. Chen and S.-J. Liu, "Chan-Chyan-Srivastava multivariable polynomials associated with a certain family of partial differential operators." Integral Transforms and Spec. Funct., vol. 22, no. 11, pp. 861-865, 2011, doi: 10.1080/10652469.2010.541158.

[6] K.-Y. Chen, S.-J. Liu, and H. Srivastava, "Some new results for the Lagrange polynomials in several variables." Anziam J., vol. 49, pp. 243-258, 2007.

[7] E. Erkus-Duman, "Matrix extensions of polynomials in several variables." Util. Math., vol. 85, pp. 161-180, 2011.

[8] E. Erkus-Duman, A. Altin, and R. Aktas, "Miscellaneous properties of some multivariable polynomials." Math. Comput. Model., vol. 54, no. 9-10, pp. 1875-1885, 2011, doi: 10.1016/j.mcm.2011.04.010.

[9] E. Erkus-Duman and O. Duman, "Integral-type generalizations of operators obtained from certain multivariable polynomials.” Calcolo, vol. 45, no. 1, pp. 53-67, 2008, doi: 10.1007/s10092-0080143-6.

[10] E. Erkus-Duman and O. Duman, "Statistical approximation properties of high order operators constructed with the Chan-Chyan-Srivastava polynomials." Appl. Math. and Comp., vol. 218, no. 5, pp. 1927-1933, 2011, doi: 10.1016/j.amc.2011.07.004.

[11] E. Erkus-Duman, O. Duman, and H. Srivastava, "Statistical approximation of certain positive linear operators constructed by means of the Chan-Chyan-Srivastava polynomials." Appl. Math. and Comp., vol. 182, no. 1, pp. 213-222, 2006, doi: 10.1016/j.amc.2006.01.090.

[12] S. Gaboury, M. Özarslan, and T. R., "Some bilateral generating functions involving the ChanChyan-Srivastava polynomials and some general classes of multivariable polynomials," Commun. Korean Math. Soc., vol. 28, no. 4, pp. 783-797, 2013, doi: 10.4134/CKMS.2013.28.4.783.

[13] B. González, J. Matera, and H. Srivastava, "Some q-generating functions and associated generalized hypergeometric polynomials." Math. Comput. Model., vol. 34, pp. 133-175, 2001, doi: 10.1016/S0895-7177(01)00053-X.

[14] C. Kaanoglu and M. Özarslan, "New Families of Generating Functions for Certain Class of Three-Variable Polynomials." Appl. Math. and Comp, vol. 218, pp. 836-842, 2011, doi: 10.1016/j.amc.2011.01.083.

[15] C. Kaanoglu and M. Özarslan, "Two-sided generating functions for certain class of r-variable polynomials." Math. Comput. Model., vol. 54, pp. 625-631, 2011, doi: 10.1016/j.mcm.2011.03.006.

[16] C. Kaanoglu and M. Özarslan, "Two-parameter Srivastava polynomials and several series identities." Adv. Diff. Eq., vol. 81, 2013, doi: 10.1186/1687-1847-2013-81. 
[17] S.-J. Liu, C.-J. Chyan, H.-C. Lu, and H. Srivastava, "Multiple Integral Representations for Some Families of Hypergeometric and Other Polynomials." Math. Comput. Model., vol. 54, pp. 1420 1427, 2011, doi: 10.1016/j.mcm.2011.04.013.

[18] S.-J. Liu, C.-J. Chyan, H.-C. Lu, and H. Srivastava, "Bilateral generating functions for the ChanChyan-Srivastava polynomials and the generalized Lauricella functions." Integral Transforms and Spec. Funct., vol. 23, pp. 539-549, 2012, doi: 10.1080/10652469.2011.610152.

[19] M. Özarslan, "Some families of generating functions for the extended Srivastava polynomials." Appl. Math. and Comp, vol. 218, pp. 959-964, 2011, doi: 10.1016/j.amc.2011.01.064.

[20] E. Özergin, M. Özarslan, and H. Srivastava, "Some families of generating functions for a class of bivariate polynomials." Math. Comput. Modelling, vol. 50, no. 7-8, pp. 1113-1120, 2009, doi: 10.1016/j.mcm.2009.05.030.

[21] H. Srivastava, "A contour integral involving Fox's $H$-function.” Indian J. Math., vol. 14, pp. 1-6, 1972.

[22] H. Srivastava, K. Nisar, and K. M.A., "Some Umbral Calculus Presentations of the Chan-ChyanSrivastava Polynomials and the Erkus-Srivastava Polynomials," Proyecciones J. Math., vol. 33, no. 1, pp. 77-90, 2014, doi: 10.4067/S0716-09172014000100006.

[23] H. Srivastava, M. Özarslan, and C. Kaanoğlu, "Some families of generating functions for a certain class of three-variable polynomials." Integral Transforms and Spec. Funct., vol. 21, no. 12, pp. 885-896, 2010, doi: 10.1080/10652469.2010.481439.

Authors' addresses

M.A. Özarslan

Eastern Mediterranean University, Faculty of Arts and Sciences, Department of Mathematics, Gazimagusa, Mersin 10, Turkey

E-mail address: mehmetali.ozarslan@emu.edu.tr

R. Srivastava

University of Victoria, Department of Mathematics and Statistics, Victoria, British Columbia V8W 3R4, Canada

E-mail address: rekhas@math.uvic.ca

C. Kaanoğlu

Cyprus International University, Faculty of Engineering, Nicosia, Mersin 10, Turkey

E-mail address: kaanoglu@ciu.edu.tr 